general morphological, physiological features of skeletal muscle and its general pathological reactions. In addition to discussions of biopsy handling and staining techniques, there is also a brief section illustrating common histological and ultrastructural artifacts. The remainder of the chapters in this section illustrate and discuss the normal and abnormal cellular and subcellular components of skeletal muscle in some detail. The second major section describes the pathology of skeletal muscle on a disease-by-disease basis.

The division of the text into two sections has an invaluable didactic advantage for the diagnostician. Muscle diseases rarely have pathognomonic single abnormalities, and usually it is a suite of abnormalities that points to the disease in question. The initial discussion of abnormalities on a structure-by-structure basis is therefore useful in shaping the differential diagnosis and guiding discussion with the referring clinician. Where a disease is defined by a single morphological feature (e.g. myopathy with cylindrical spirals) there is occasionally some redundancy. The discussion is skeptical in tone but insightful. I sometimes found myself wishing for more discussion of the molecular aspects of some disease entities, but in general the text is concise, and the references carefully chosen. There is considerable historical depth in some areas as well: these features strongly and positively reflect on the clinical experience of both authors.

Probably the strongest feature of the book is the wealth of superb illustrations of ultrastructural and light microscopic features. There are over 100 beautifully printed color plates, and over 600 black and white photographs. These are carefully and liberally distributed throughout the text. The variety and quality of these illustrations alone ensure that this book will remain an important diagnostic guide for some time to come. I would recommend this reference to anyone interested in morphological aspects of skeletal muscle disease, whether resident, fellow or practicing clinician.

Patrick Shannon

Toronto, Ontario

\section{Cerebral Ischemia: Molecular and Cellular} Pathophysiology. 1st Edition. 1999. Edited by Wolfgang Walz. Published by Humana Press Inc. 278 pages. C $\$ 181.25$ approx.

Cerebral Ischemia: Molecular and Cellular Pathophysiology is presented as a text that will provide pertinent and up-to-date information to a variety of interested readers. The contents of the book are laid out in a manner that targets health care professionals who are already seasoned in this field. The book begins with a detailed overview of the differing mechanisms leading to ischemic damage and the resultant cellular and neuronal death associated with it. The remainder of the text is divided into two sections, factors in the brain microenvironment and cellular changes associated with ischemic episodes.

The section "Factors in the Brain Microenvironment" is divided into subsections which address issues of electrophysiology, edema formation, calcium overload, oxygen radicals and initiators of inflammatory responses. These subsections, in a succinct manner, contribute to the overall theme of the book by successively covering individual but related factors in the brain microenvironment. The format is a compilation of scientific review articles with the underlying message of how neurons die during and following ischemic episodes. A reader with experience in this field will be able to tie the separate issues presented into a cohesive whole. However, due to lapses in clarity and a lack of illustrations to demonstrate the inter-relationship among the many factors covered, this series of subsections will not allow the less informed reader to gain a comprehensive understanding of the central message.

The section, "Cellular Changes", is also divided into subsections, with a similar format to the previous one. This section addresses the issues of altered gene expression, the debate of necrosis versus apoptosis, gliosis and phagocytosis, with a central message of altered cellular performance in the face of an ischemic episode. It flows from topic to topic with a more developed sense of clarity, but remains a compilation of review articles. Once again an informed reader with the ability to make links among the subjects covered separately will benefit from the information provided. However, a less informed reader will be left with the sense that they have not understood the central message.

To conclude, this is a very useful book for the experienced stroke physician and the educated basic scientist/student of cerebral ischemia research. It is, therefore, highly recommended to these groups as a source of information, as an instructional tool and to stimulate insight and new hypothesis to define future experimentation.

Daniel T. Warren and Alastair M. Buchan Calgary, Alberta

Handbook of Ataxia Disorders. 2000. Edited by Thomas Klockgether. Published by Marcel Dekker, Inc., New York. 688 pages. C\$316.05 approx.

This multi-authored volume addresses basic and clinical aspects relating to the broad spectrum of cerebellar disorders. It is directed primarily at the clinician who deals with these patients. Although multi-authored, the editor has been able to minimize style differences between chapters by applying a uniform format throughout the volume. Hence, each chapter begins with an outline of its contents, each organized in a similar fashion. A curious oversight is in the first chapter, in which the references are listed differently from elsewhere in the book.

Initial chapters deal with the functional architecture and physiology of the cerebellum, and with the history of ataxia research. Neurology residents will find the brief chapter summarizing a clinical approach to cerebellar dysfunction to be particularly useful. Individual chapters are devoted to each of the major subtypes of cerebellar disease, with each chapter including sections on epidemiology, molecular pathogenesis, neuropathology, and clinical features. Overlap between chapters, although inevitable to some degree, has been kept to a minimum. Each of the autosomal dominant spinocerebellar ataxias (types 1-7 and 10) are dealt with separately, with ample information to instruct the reader on the differences and similarities, in terms of both clinical and DNA abnormalities. The autosomal recessive ataxias are dealt with in a similar fashion, with scholarly chapters on Friedreich's ataxia, ataxia-telangiectasia, Refsum's disease, and other recessive disorders. From a Canadian standpoint, it was refreshing to see a chapter dedicated to the Charlevoix-Saguenay form of autosomal recessive spastic ataxia. Other chapters include one on prion diseases associated with ataxia, sufficiently current that it includes the important observation that ataxia is an early feature in almost all 
patients with new variant Creutzfeldt-Jakob disease. A scholarly discussion of multiple system atrophy includes useful "red flags" to keep in mind when considering the possibility of this syndrome occurring in the setting of parkinsonism.

The authors should be complemented on producing such an allinclusive volume. It is a bit large to be called a "handbook", but is a book that should be found on the shelves of any clinician that has more than a passing interest in these disorders. Neurology trainees will find it particularly useful as they are making their way through this often confusing territory.

Wayne Martin Edmonton, Alberta

Neurological Eponyms. 2000. By P.J. Koelhler, G.W. Bruyn, J.M.S. Pearce (eds). Published by New York, Oxford University Press. C\$ 87.95 approx.

This is a collection of 55 essays written by 51 prominent neurologists and other neuroscientists. They are grouped under the headings Structures and Processes; Symptoms and Signs; Reflexes and other Tests; Syndromes; and Diseases and Defects. Almost every one is illustrated with a portrait of its subject and most have additional diagrams of the entity linked with his name - no women are represented, not even Mme Dejerine (but then again, nor is her husband). Each chapter runs to 2000 words or so, and referencing is very adequate.

The work is an expansion of a shorter set of essays on the founders of the neurological examination, published in Holland in 1995, and will be a welcome addition to the library of those who like to achieve some mental closure through understanding of the circumstances under which the aspect of neurology in question was first defined, and through attainment of some familiarity with details of its progenitor. The biographies are agreeably succinct while being adequately detailed, but most who buy the book will have much of this information already. It is the critical evaluations of the phenomena, in each case presenting the relevant data and analyzing them in fair but not excessive depth, that make the book most appealing.

There are a few issues on which a picky reviewer might take issue - did Sir Henry Head truly report that gall-bladder pain refers to the left shoulder? Was not the most useful sign described by Jules Froment (the 'circle sign') that which assists in the diagnosis of anterior interosseous palsy rather than the clumsy signe de journal for ulnar nerve palsy? How does the cerebellum displace downwards within the fourth ventricle in the Chiari malformation? How many of the patients of John Norris with vertebral artery dissections following chiropractic manipulation developed a Wallenberg syndrome? But this book contains only a few such small infelicities, while it illuminates our discipline by presenting these named phenomena both in their human and in their neurological contexts. Correctly, the editors allege the resurgence of eponyms and defend their continued use in response to the increasing prolixity of scientific nomenclature. In employing them, we honour their discoverer, embellish our understanding and smooth communication.

Neurological Eponyms contains more than its title suggests. It would be a perfect gift for a neurologist friend, for a succeeding resident and for your departmental library. And also for yourself.

William Pryse-Phillips St John's, Newfoundland
EPILEPSY Surgery. 2001. 2nd Edition. By H.O. Lüders and Y.G. Comair. Published by Lippincott Williams \& Wilkins. 1060 pages. C $\$ 292.53$ approx.

Many pearls are contained within this book but one has to shuck a lot of oysters to get at them. It is less a textbook than a collection of essays by well-recognized authorities active in the surgical treatment of patients with medically intractable epilepsy. It is a big and broad and heavy book comprised of 180 chapters grouped into 18 sections and running to over a thousand pages. It could have benefited from the editors'blue pencil to condense and abridge some of the chapters dealing with the same or similar topics so as to present a more cohesive treatise on epilepsy surgery. These minor irritants apart, the book is encyclopedic, authoritative, and entirely up-to-date. Especially welcome to this reader is a discussion of the contributions of Fedor Krause and Otfrid Foerster to the surgical treatment of epilepsy in the history section of the book. This section is followed by an overview of epilepsy surgery. The meat of the book is entered in section three where epileptic syndromes are addressed. Especially noteworthy in this section is the discussion on Rasmussen's syndrome. A short section on presurgical evaluation precedes a more extensive discussion on structural and functional neuroimaging. Generally speaking the quality of the reproduced images is satisfactory, and the quality and detail of the images in the chapter on imaging of the cerebral cortex are stunning. The architectural planning of a monitoring unit, various modes of detection of epileptogenic foci, and discussions of electroencephalography and magnetoencephalography precede a section on neuropsychology and psychiatry. This is followed by four chapters on sodium amobarbital testing. By this time $60 \%$ of the book has been covered with nary a mention of surgery. This is remedied in the following 250 pages where invasive procedures for electroencephalography with foramen ovale, epidural, subdural, and depth electrodes, alone or in combination, are addressed. There follows an extensive discussion of therapeutic surgical techniques for the resection of epileptogenic foci and lesions in specific parts of the brain. (Parenthetically, the surgical treatment of frontal lobe epilepsy is addressed in a chapter that has five more authors than the number of its pages). There is an especially strong chapter on the selection of surgical procedures for patients with temporal lobe epilepsy. Due importance is given to structural brain lesions such as cortical dysplasias, tumors and vascular lesions as a source of epileptic seizures. Other procedures, such as hemispherectomy in its various guises, callosotomy, and vagal nerve stimulation, an especially timely topic, are addressed in detail. Most of the remainder of the book deals with outcome and the management of surgical failures. The book ends with seven appendices addressing a variety of topics, some already dealt with in the body of the book. Throughout the book, special attention is given to the pediatric population in terms of investigation, surgical planning, and surgical techniques.

The overall impression then is of a very timely, highly authoritative and comprehensive book that will serve as a useful reference for anyone interested in the field, from the junior resident to the most experienced epileptologist.

Richard Leblanc Montreal, Ontario 